\title{
In Vivo Effects of Cagaita (Eugenia dysenterica, DC.) Leaf Extracts on Diarrhea Treatment
}

\author{
T. B. Lima, ${ }^{1}$ O. N. Silva, ${ }^{1,2}$ L. P. Silva, ${ }^{3}$ T. L. Rocha, ${ }^{3}$ M. F. Grossi-de-Sá, ${ }^{3}$ O. L. Franco,, 2 \\ and $\mathrm{E}$. Leonardecz \\ ${ }^{1}$ Centro de Análises Proteômicas e Bioquímicas, Programa de Pós-Graduação em Ciências Genômicas e Biotecnologia, \\ Universidade Católica de Brasília, SGAN Quadra 916, Modulo B, Avenue W5, 70 790-160 Brasília, DF, Brazil \\ ${ }^{2}$ Programa de Pós-graduação em Ciências Biológicas (Imunologia/Genética e biotecnologia), Universidade Federal de Juiz de Fora, \\ 36036-330 Juiz de Fora, MG, Brazil \\ ${ }^{3}$ Embrapa Recursos Genéticos e Biotecnologia, Laboratório de Espectrometria de Massa e Laboratório de Interação Molecular \\ Planta-Praga, PqEB, Avenue W5 Norte, 70770-900 Brasília-DF, Brazil \\ ${ }^{4}$ Universidade de Brasília, Campus Universitário de Planaltina Vila Nossa Senhora de Fátima, 73300-000 Brasília, DF, Brazil
}

Correspondence should be addressed to O. L. Franco, ocfranco@gmail.com

Received 5 May 2010; Accepted 1 July 2010

Copyright ( $) 2011$ T. B. Lima et al. This is an open access article distributed under the Creative Commons Attribution License, which permits unrestricted use, distribution, and reproduction in any medium, provided the original work is properly cited.

Eugenia dysenterica is a plant typically found in the Cerrado biome and commonly used in popular medicine due to its pharmacological properties, which include antidiarrheal, skin healing, and antimicrobial activities. The effects of ethanolic extract, aqueous extract and infusion of $E$. dysenterica leaves on intestinal motility and antidiarrheal activity were evaluated using ricin oilinduced diarrhea in rats. At doses of 400 and $800 \mathrm{mg} \cdot \mathrm{Kg}^{-1}$, the ethanolic extract decreased intestinal motility while the other extracts showed no significant effects. Moreover, serum levels of chloride, magnesium, and phosphorus were also measured in rats. Histopathologic and enzymatic analyses were also performed to investigate any toxic effect. Animals treated with infusion, ethanolic extract, ricin oil, and loperamide presented morphological alterations in the small intestine, such as mucosa lesion, epithelial layer damage, and partial loss and/or morphological change of villi. Furthermore, the liver showed congestion and hydropic degeneration. Serum levels of alanine aminotransferase increased significantly in all treatments, but none rose above reference values. In summary, our results suggest that compounds present in leaves of E. dysenterica may have therapeutic benefits on recovery from diarrhea despite their toxic effects.

\section{Introduction}

Diarrhea is responsible for deaths in adults and especially children all over the world, particularly in developing countries $[1,2]$. In general, chronic diarrhea can develop from congenital problems, neoplastic syndrome, human immunodeficiency virus (HIV), adverse effects of medications, and, most commonly, is caused by gastrointestinal infections including viral/bacterial gastroenteritis $[3,4]$. In general, patients with uncontrolled diarrhea are at increased risk of dehydration, electrolyte imbalance, skin breakdown, and fatigue. In many cases the treatments utilized are oral rehydration therapy (ORT) and pharmacological intervention, including antibiotics and antidiarrheal drugs, which cause a pronounced effect on gut motility, thus decreasing intestinal transit $[5,6]$. Currently, the search for antidiarrheal therapies should include allopathic, homeopathic, and alternative medical approaches that have demonstrated their ability to reduce the duration and severity of diarrhea in children in developing countries [7].

In view of a number of disease properties including epidemiology, etiological agents, and changeable clinical manifestations depending on the region, different strategies have been applied to control diarrhea [8]. In order to reduce infections caused by bacterial pathogens, the Diarrheal Disease Control program created in 1978 by the World Health Organization (WHO) puts special emphasis on the use of traditional (folk) medicines in the control and 
management of diarrhea [9]. The regional Western Pacific Office of the WHO included diarrhea as a target disease [10]. Currently, screening of biological products and phytochemical extracts has been encouraged to obtain isolated plant biomolecules with antidiarrheal activity, including various plant families such as Liliaceae [11], Rubiaceae [12], Meliaceae [13], Fabaceae [14], Myrtaceae [15], and several others.

Eugenia dysenterica DC., known as "cagaita", is a fruitbearing tree of the Myrtaceae family native to the Brazilian Cerrado (upland savannah). This plant is widely used by the population in the treatment of various diseases; tea from its leaves is used to treat diarrhea, diabetes, and jaundice; tea from its flowers is used to treat kidney and bladder infections; its fruit is used as a laxative [16]. Considering that cagaita leaf tea is the main way in which this plant is used as a popular alternative medicine in Latin America, the present study was conducted to evaluate the possible antidiarrheal effect of the leaves of E. dysenterica, analyzing antimotility and antisecretory actions. Furthermore, the possible toxic effects were evaluated in a rat model by histopathological analysis.

\section{Material and Methods}

2.1. Plant Material and Extract Preparation. Leaves from E. dysenterica were collected in the Cerrado biome, from an area of upland savannah surrounding Brasilia-DF, Brazil. Leaves were dried at $30^{\circ} \mathrm{C}$ in a circulating air oven. One hundred grams of leaf powder was subjected to sequential extraction using solvents of increased polarity to afford dry extracts of hexane $100 \%$ in a proportion of $3: 1(\mathrm{w} / \mathrm{v})$, ethanol $100 \%$ in a proportion of $3: 1(\mathrm{w} / \mathrm{v})$, and deionized water $(1: 1)$ to give hexanic, ethanolic, and aqueous extracts [16]. The solvent of ethanolic extract was evaporated under vacuum in a rotary-evaporator at $45^{\circ} \mathrm{C}$ and further stored at $24^{\circ} \mathrm{C}$. Aqueous extract was lyophilized and stored at $-20^{\circ} \mathrm{C}$. The powdered leaves (100 g) were also macerated and extracted with deionized water for 24 hours at $40^{\circ} \mathrm{C}$ under $120 \mathrm{rpm}$ agitation. After cooling, the resulting extract was filtered through $8 \mu \mathrm{m}$ filter paper and lyophilized. For bioactivity investigations, all extracts were soluble in distilled water. None difficulty in the extract solubilization was observed. Percentage yields of the hexanic, ethanolic, and aqueous extracts and of the infusion were, respectively, 8.78\%, 22.1\%, $22.4 \%$, and $20.0 \%$ of dry weight.

2.2. Animals. Male adult Wistar rats (Rattus norvegicus) weighing 280-300 g were acquired at the Biotery of the Universidade Católica de Brasília. All animals were used after 1 week of acclimatization (temperature $23 \pm 2{ }^{\circ} \mathrm{C}$ humidity $60 \%$ ) and had free access to water and food. The study was approved by the Animal Use Committee (CEUA) at the Institute of Biological Sciences, University of Brasilia. Project number 0177-07 was approved on 27.03.07 under the name "Activity of drugs in the presence or absence of nanocapsules and/or magnetic fluids in the treatment of several diseases in experimental animal models". The experiments reported here comply with ethical procedures with investigated animals [17].

2.3. Assessment of Gastrointestinal Propulsion of Charcoal Meal. Evaluation of antidiarrheal effects of E. dysenterica was conducted according to the method described by Abdullahi et al. [18]. Forty rats were separated in five groups of eight animals. The positive control group received loperamide orally, at the dose of $2 \mathrm{mg} \cdot \mathrm{kg}^{-1}$ and the negative group received $1 \mathrm{~mL}$ of water; test groups received the infusion or aqueous extract at doses of $800 \mathrm{mg} \cdot \mathrm{kg}^{-1}$ or ethanolic extract at doses of $400 \mathrm{mg} \cdot \mathrm{kg}^{-1}$ resuspended in $1 \mathrm{~mL}$ of water. This procedure was determined according to Sagar et al. [19], Mbagwu and Adeyemi [20], Ojewole et al. [21], Mujumdar et al. [22], and Afroz et al. [23] with minor modifications. Test animals were starved for 12 hours prior to the experiment, but consumed water ad libitum. Ten min later, animals from each group were fed on $1 \mathrm{~mL}$ of charcoal meal (3\% suspension of deactivated charcoal in $0.5 \%$ aqueous methylcellulose). Thirty minutes after the administration of charcoal meal, the animals from each group were killed by using $\mathrm{CO}_{2}$, and intestine length (pyloric sphincter to caecum) was measured, as well as the distance along which the charcoal moved as a fraction of that length, in accordance with the procedure described by Nwafor et al. [11]. The mean value for each group was calculated and results obtained in the control and test groups were compared. Percentage inhibitions of intestinal motility were calculated by using the equation (negative control mean-treated (test) mean)/negative control mean $\times 100$.

2.4. Evaluation of Serum Levels. Analyses of ionic absorption and acute and subchronic toxicity after ricin oil-induced diarrhea were conducted according to the method described by Sagar et al. [19] with some modifications. Rats were divided into six groups of eight animals; diarrhea was induced by administering $1 \mathrm{ml}$ of ricin oil orally using an intragastric tube. Thirty minutes after the administration of ricin oil, the animals received the treatments. The positive control group received loperamide orally, at the dose of $2 \mathrm{mg} \cdot \mathrm{kg}^{-1}$. The negative control was divided in to two groups including animals treated orally with $1 \mathrm{~mL}$ of ricin oil or $0.2 \mathrm{~mL}$ of water using an intragastric tube. Test groups received the infusion or aqueous extracts at doses of $800 \mathrm{mg} \cdot \mathrm{kg}^{-1}$ or the ethanolic extract at the dose of $400 \mathrm{mg} \cdot \mathrm{kg}^{-1}$, resuspended in $0.2 \mathrm{~mL}$ of water. This assay was conducted for twenty-two days, after which the blood to be used for the evaluation of serum levels was collected from all animals. After different drug administration periods (no administration-T0, one dose-T1, repeated doses for 7 days_-T2, and repeated doses for 14 days-T3), $1 \mathrm{~mL}$ of blood was collected by heart puncture, without anticoagulant substances, in order to obtain serum samples, and further analyzed according to several biochemical variables such as chloride, magnesium, phosphorus, and alanine aminotransferase by using commercial kits (Labtest Diagnostic S.A., Lagoa Santa-MG, Brazil). 


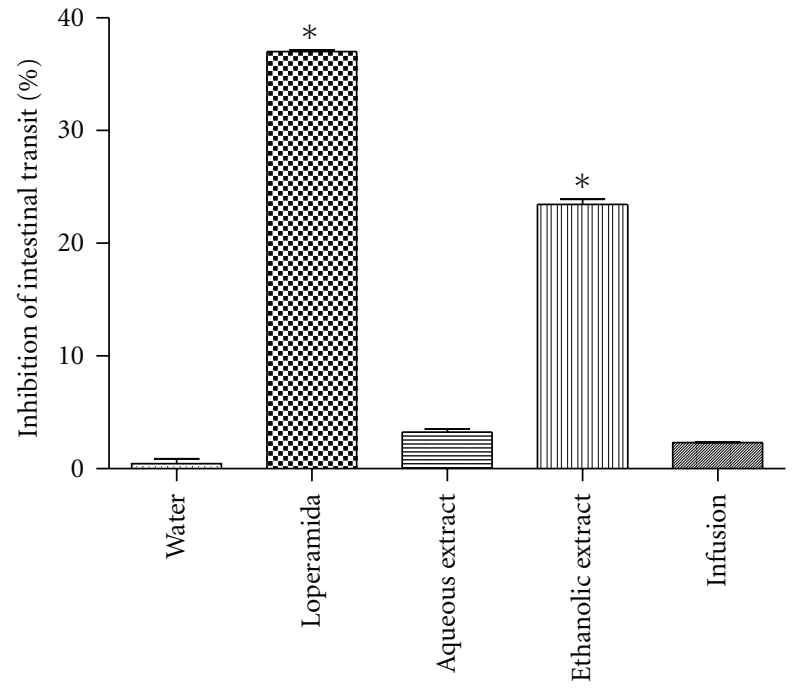

Figure 1: Evaluation of antimotility activity after administration of infusion and aqueous extract at the dose of $800 \mathrm{mg} \cdot \mathrm{kg}^{-1}$ and ethanolic extract at the dose of $400 \mathrm{mg} \cdot \mathrm{kg}^{-1}$. The positive control group received loperamide at the dose of $2 \mathrm{mg} \cdot \mathrm{kg}^{-1}$, and the negative control group was given $1 \mathrm{~mL}$ of water orally. Vertical bars represent standard error. Asterisks indicate statistical difference from control $(P \leq .05)$ by Dunnett test.

2.5. Histopathological Analysis. All animals that survived 22 days of treatment were submitted to laparotomy for removal of the small intestine and liver. These tissues were fixed in $10 \%$ neutral buffered formalin. The fixed samples were dehydrated in an ascending series of ethanol, cleared in methyl benzoate, and embedded in paraffin wax. Four- $\mu \mathrm{m}-$ thick sections were obtained using a microtome and stained with hematoxylin and eosin. The photomicrographs were taken by digital camera (AxioCam MRc 5, Carl Zeiss) driven by software Axio Vision 4.6.3. (Carl Zeiss) coupled with an optical microscope (Carl Zeiss). Tissue lesion in the intestine was classified by the occurrence of villi alterations and ulceration or inflammatory process. Tissue lesion of the liver was determined by the presence of congestion, hydropic and fatty degeneration, inflammatory infiltrate, and necrosis.

2.6. Statistical Analysis. Results were expressed as means \pm $\mathrm{SD}$ of four separate experiments. Data from all assays were subjected to a Shapiro-Wilk test of normality statistical analysis of data obtained was performed using one-way analysis of variance (ANOVA), and the differences among group means were analyzed using Dunnett's multiple comparisons test. Analysis was performed using BioEstat software (v. 5.0). Values were held to be significant if $P<.05$.

\section{Results}

3.1. Effect of Leaf Samples of E. dysenterica on Gastrointestinal Transit of Charcoal Meal. Initially, experiments carried out on intestinal transit after charcoal meal administrations were conduced. The ethanolic extract at the dose of $400 \mathrm{mg} \cdot \mathrm{kg}^{-1}$

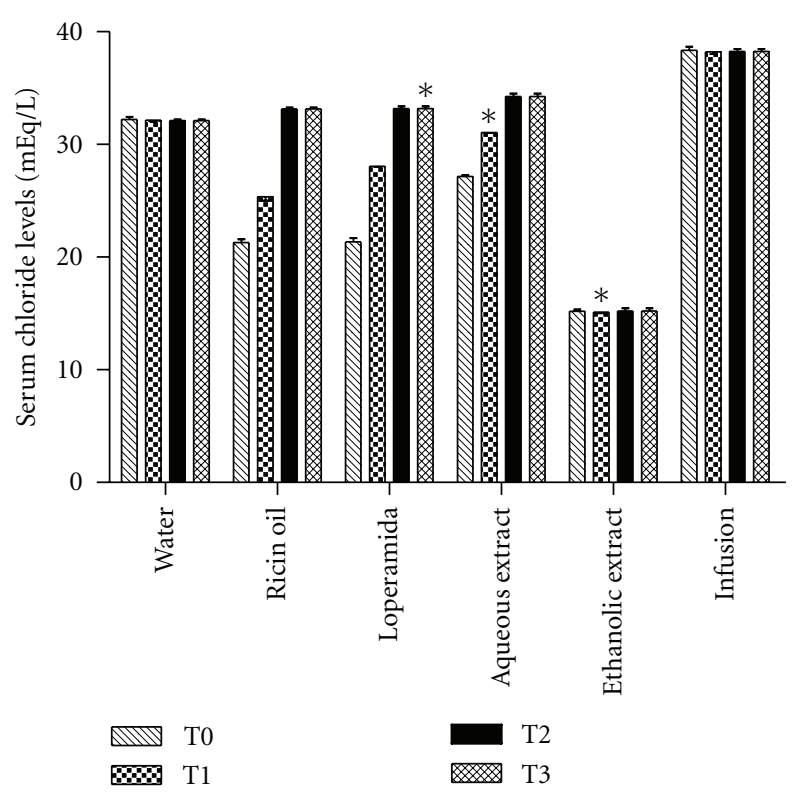

FIGURE 2: Analysis of the antisecretory effect of infusion, ethanolic and aqueous extract of E. dysenterica utilizing as parameters alterations in serum chloride levels at different periods: no treatments (T0), after one dose (T1), seven-day dose (T2) and 14day dose (T3). Negative control group received $1 \mathrm{~mL}$ of water and ricin oil $\left(10 \mathrm{mg} \cdot \mathrm{kg}^{-1}\right)$. Positive control group received loperamide $\left(2 \mathrm{mg} \cdot \mathrm{kg}^{-1}\right)$ and experimental groups received infusion and aqueous extract at the dose of $800 \mathrm{mg} \cdot \mathrm{kg}^{-1}$ and ethanolic extract at the dose of $400 \mathrm{mg} \cdot \mathrm{kg}^{-1}$. Vertical bars represent standard error. Asterisks indicate statistical difference from control $(P \leq .05)$ by Dunnett test.

and loperamide at the dose of $2 \mathrm{mg} \cdot \mathrm{kg}^{-1}$ significantly inhibited $(P<.05)$ intestinal transit in rats by $24 \%$ and $37 \%$, respectively (Figure 1). Nevertheless, aqueous extract and infusion did not cause any effect on intestinal transit, showing similar data to those of the negative control (water).

\subsection{Evaluation of Ion Absorption after Ricin Oil-Induced} Diarrhea. In the present study, diarrhea was induced with ricin oil, and the antidiarrheal that effect produced by samples was analyzed utilizing chloride, magnesium, and phosphorus levels at different periods. In animals treated with loperamide, levels of chloride increased by $41.2 \%$ $(P<.05)$ (Figure 2). Animals treated with the aqueous and ethanolic extracts also showed a significant increase in levels of chloride, by $20.0 \%$ and $30.0 \%$, respectively $(P<.05)$. The other treatments showed no significant variation in serum levels of chloride with a consecutive dose of $800 \mathrm{mg} \cdot \mathrm{kg}^{-1}$ for a period of 14 days (Figure 2).

Analysis of phosphorus concentration showed initial decrease in animals' serum levels when treated with ricin oil $(23 \%)$, loperamide $(33 \%)$, aqueous extract $(10 \%)$, ethanolic extract (18\%), and infusion (33\%) (Figure 3). Phosphorous in these groups decreased significantly $(P<.05)$ after the administration of only one dose. Continued use could lead to hyperphosphatemia in animals treated with ricin oil $(80.5 \%)$, loperamide $(68.4 \%)$, aqueous extract $(85.4 \%)$, 


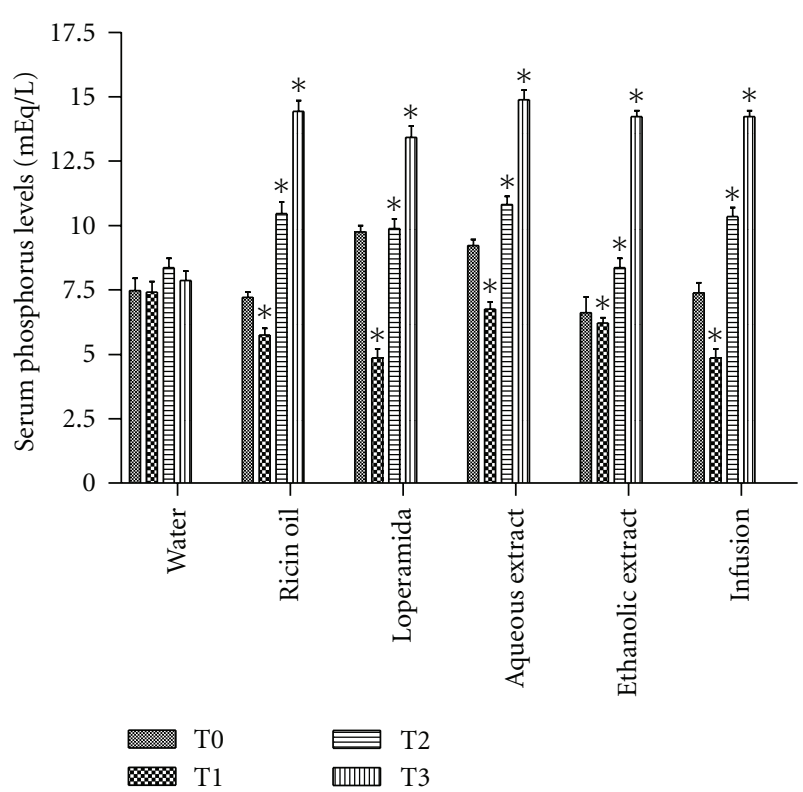

FIgURE 3: Analysis of antisecretory effect of infusion, ethanolic and aqueous extracts of E. dysenterica utilizing as parameters alterations in serum phosphorus levels at different periods: no treatments (T0), after one dose (T1), seven-day dose (T2), and 14day dose (T3). Negative control group received $1 \mathrm{~mL}$ of water and ricin oil $\left(10 \mathrm{mg} \cdot \mathrm{kg}^{-1}\right)$. Positive control group received loperamide $\left(2 \mathrm{mg} \cdot \mathrm{kg}^{-1}\right)$, and experimental groups received infusion and aqueous extract at the dose of $800 \mathrm{mg} \cdot \mathrm{kg}^{-1}$ and ethanolic extract at the dose of $400 \mathrm{mg} \cdot \mathrm{kg}^{-1}$. Vertical bars represent standard error. Asterisks indicate statistical difference from control $(P \leq .05)$ by Dunnett test.

ethanolic extract $(75.7 \%)$, and infusion $(75.7 \%)(P<.05)$ (Figure 3).

Magnesium level analyses of serum were altered in rats treated with ricin oil (165\%), loperamide $(82.8 \%)$, aqueous extract $(58.8 \%)$, ethanolic extract $(53 \%)$, and infusion $(82.8 \%)(P<.05)$ (Figure 4$)$. These alterations in serum magnesium levels were mainly observed after consecutive doses (T3), causing a clear increase of over 100\%. Moreover, it is important to observe that in all analyses of serum levels, the animals that received $1 \mathrm{~mL}$ of water (negative control) did not show any modifications in the concentrations of this ion.

3.3. Histopathological Analysis. The histopathological analysis of the animals, after 22 days of administration of treatments, showed significant alterations in intestine and liver tissue. In general, the small intestine evidenced the presence of an inflammatory process causedto all test groups. However, in the animals treated with infusion, it is important to note the presence of intense inflammatory infiltrate, partial loss of intestinal villi, and a complete mucosal alteration (Figure 5(b)). Animals that received ethanolic extract showed congestion and changes in the morphology of the villi (Figure 5(c)). Ulceration and intense inflammatory infiltrate were observed in animals treated with aqueous extract and ricin oil (Figures 5(d) and 5(e), resp.). Animals that received loperamide showed changes and destruction in

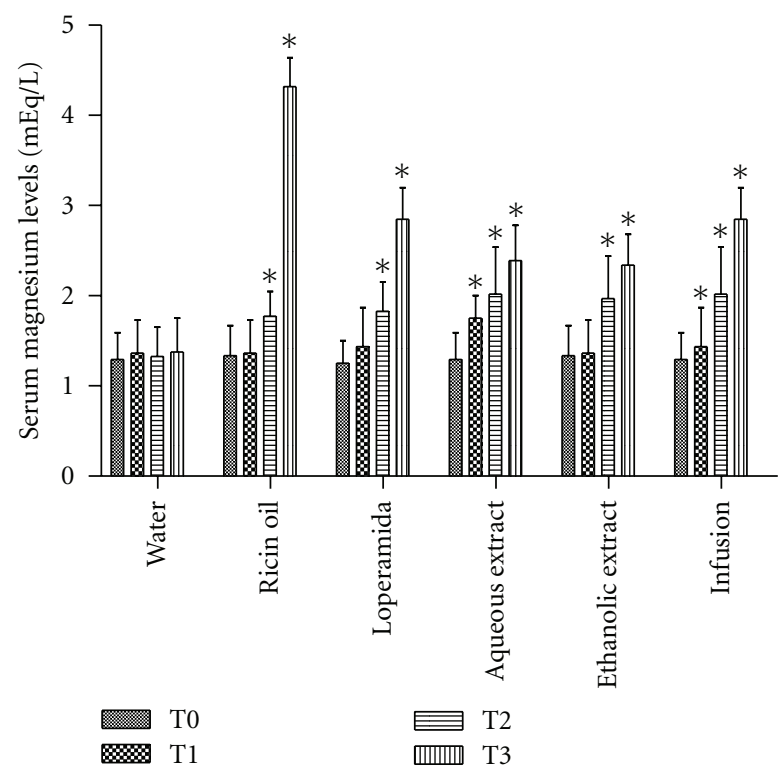

FIGURE 4: Analysis of antisecretory effect of infusion, ethanolic and aqueous extracts of E. dysenterica utilizing alterations in serum magnesium levels at different periods: no treatments (T0), after one dose (T1), seven-day dose (T2) as parameters, and 14-day dose (T3). Negative control group received $1 \mathrm{~mL}$ of water and ricin oil $\left(10 \mathrm{mg} \cdot \mathrm{kg}^{-1}\right)$. Positive control group received loperamide $\left(2 \mathrm{mg} \cdot \mathrm{kg}^{-1}\right)$, and experimental groups received infusion and aqueous extract at the dose of $800 \mathrm{mg} \cdot \mathrm{kg}^{-1}$ and ethanolic extract at the dose of $400 \mathrm{mg} \cdot \mathrm{kg}^{-1}$. Vertical bars represent standard error. Asterisks indicate statistical difference from control $(P \leq .05)$ by Dunnett test.

the morphology of the villi (Figure 5(c)). Moreover, it should be noted that aqueous extract and ricin oil caused greater damage to the intestinal tissue than to the other analyzed groups.

Histopathological analysis of the liver after infusion revealed that the sample caused congestion in the centrolobular veins, and this symptom could be associated with a congestion focus in the sinusoid capillary, hydropic degeneration, and portal/periportal oedema formation (Figure 6(b)). Similar data were observed in animals that received ricin oil, and a cluster of cords of hepatocytes was also described in association with this alteration, except for the presence of an inflammatory process and the absence of portal/periportal oedema formation in this group (Figure 6(e)). It should be emphasized that animals that received water did not show any modification (Figures 5(a) and 6(a)). Furthermore, the consecutive administration of loperamide caused the greatest liver alterations. Animals that received loperamide, ethanolic and aqueous extracts showed hydropic degeneration, sinusoidal congestion in the capillaries, and portal/periportal oedema formation (Figure 6(f)).

3.4. Analysis of Serum Alanine Aminotransferase. The levels of alanine aminotransferase (ALT) increased in animals treated with ricin oil (54\%), loperamide (54\%), and aqueous extract $(26 \%)$ (Figure 7$)$. It is noteworthy that despite 


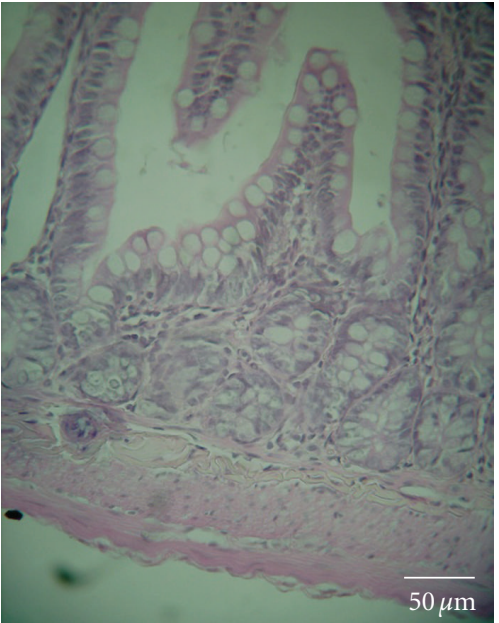

(a)

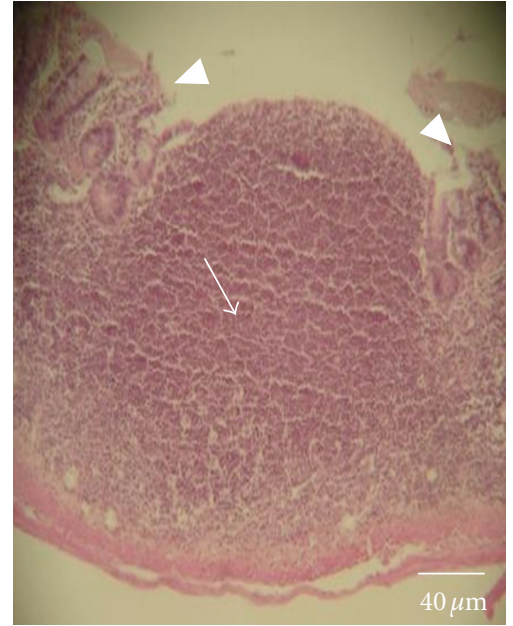

(d)

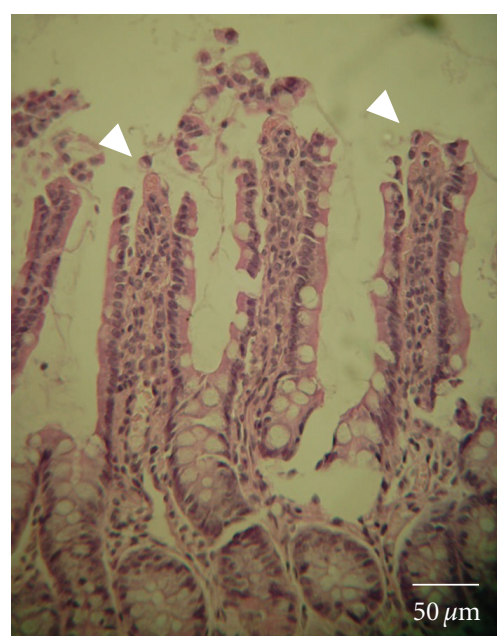

(b)

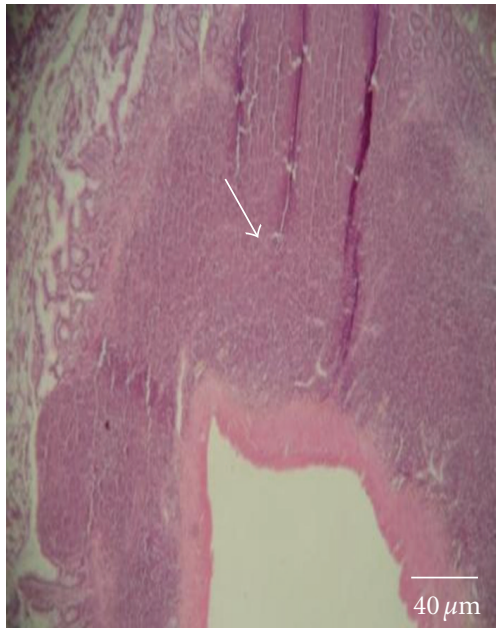

(e)

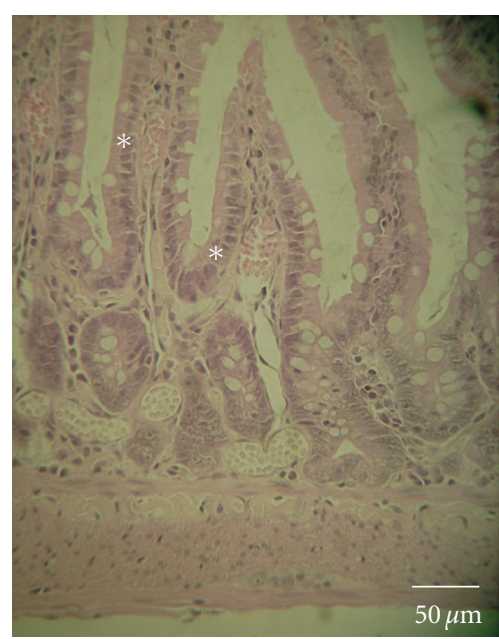

(c)

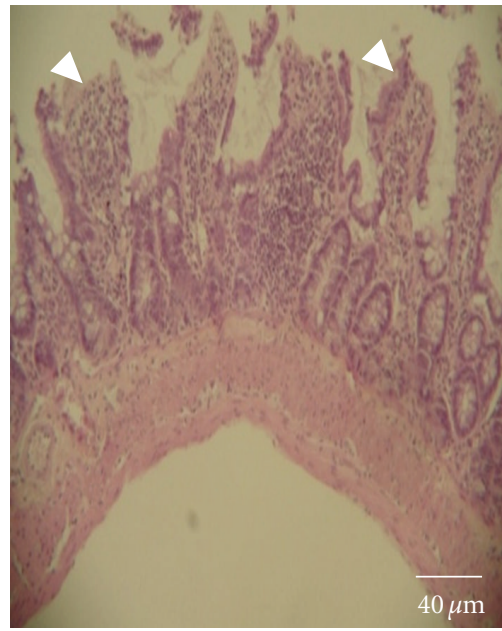

(f)

Figure 5: Small intestine sections of rats treated with water (a), infusion of leaves of E. dysenterica (b), ethanolic extract of leaves of $E$. dysenterica (c), aqueous extract of leaves of E. dysenterica (d), ricin oil (e) and loperamide (f). The arrows indicate intense mononuclear cell inflammatory infiltrate in the mucosa and submucosa; arrow tips indicate changes in villi morphology and the asterisk indicates congestion.

the increase in enzyme serum levels and tissue damage observed in the histopathological analysis, none of the treatments exceeded the reference range for this rat's enzyme, which is $35.1 \pm 13.3 \mathrm{U} / \mathrm{L}[19]$.

\section{Discussion}

Diarrhea is a consequence of innumerable pathologic conditions. Usually diarrhea is caused by altered motility and fluid accumulation in the intestine lumen, which can be occasioned by an increased secretion of electrolytes (secretory diarrhea), an enhanced ingestion of osmotic substances (osmotic diarrhea), or the presence of a virulent microorganism (infectious diarrhea) $[3,6,24]$. For this reason, in the present study, antidiarrheal properties of infusion, ethanolic and aqueous extracts of leaves of $E$. dysenterica were examined using various parameters, including intestinal motility and ionic absorption.
It was shown that the ethanolic extract of leaves of E. dysenterica significantly inhibited intestinal transit in rats, while the other two extracts were unable to do that. Similar effects are described by Sagar and collaborators [19] with the use of Lantana camara methanolic extract against neostigmine as promotility agent. Khan and collaborators in [25] showed the antidiarrheal activity from ArqueAjeeb, a mixture of Mentha arvensis, Trachyspermum ammi, and Kaphoor, which was able to inhibit intestinal transit in serotonin-induced diarrhea and PGE2-induced small intestine enteropooling. Various plant extracts are described as having antidiarrheal activity, such as aqueous extracts of Guiera senegalensis roots [26] and Byrsocarpus coccineus [27], and also methanol extracts of Xylocarpus granatum bark [12], Asparagus pubescens root [10], Dalbergia lanceolaria bark [13], and others.

Antidiarrheal activity was found in plants possessing tannins, alkaloids, saponins, flavonoids, steroids, and terpenoids 


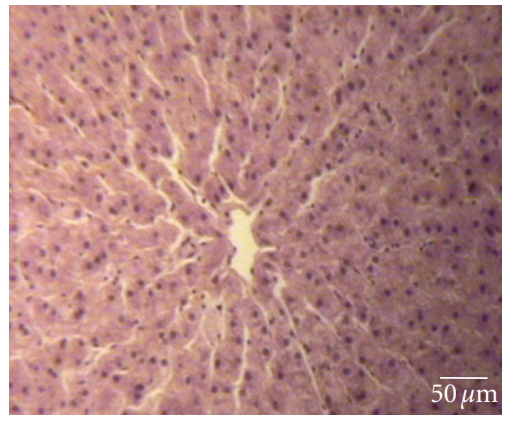

(a)

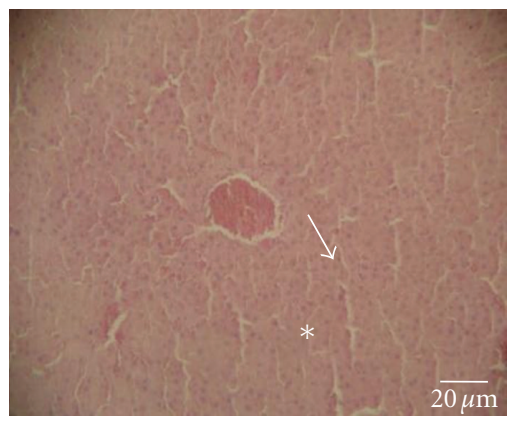

(d)

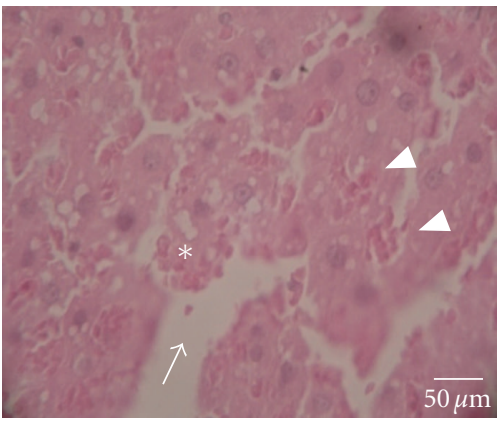

(b)

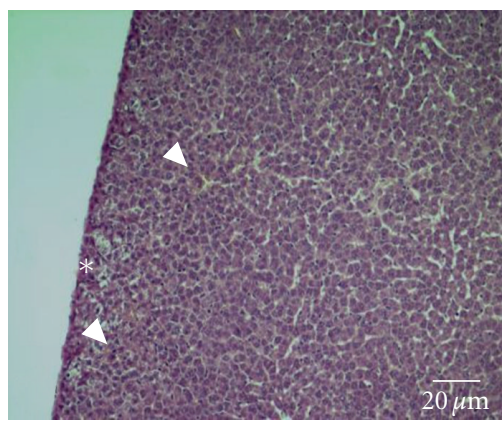

(e)

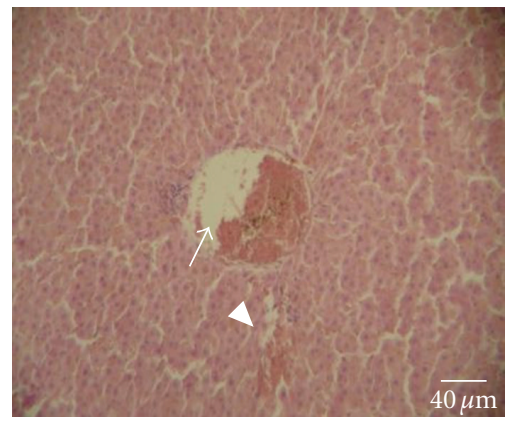

(c)

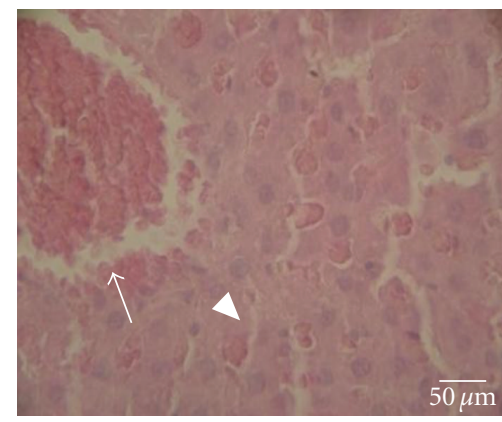

(f)

FiguRE 6: Liver sections of rats treated with water (a), infusion of leaves of E. dysenterica (b), ethanolic extract of leaves of E. dysenterica (c), aqueous extract of leaves of E. dysenterica (d), ricin oil (e) and loperamide (f), The arrows indicate congestion in the centrolobular vein, the arrow tips show congestion in the capillary sinusoids and the asterisks indicate hydropic degeneration in $b$ and fatty degeneration in $d$. Figures $\mathrm{b}$ and $\mathrm{f}$ also show portal/periportal oedema formation. Figure e present numerous inflammatory process spots.

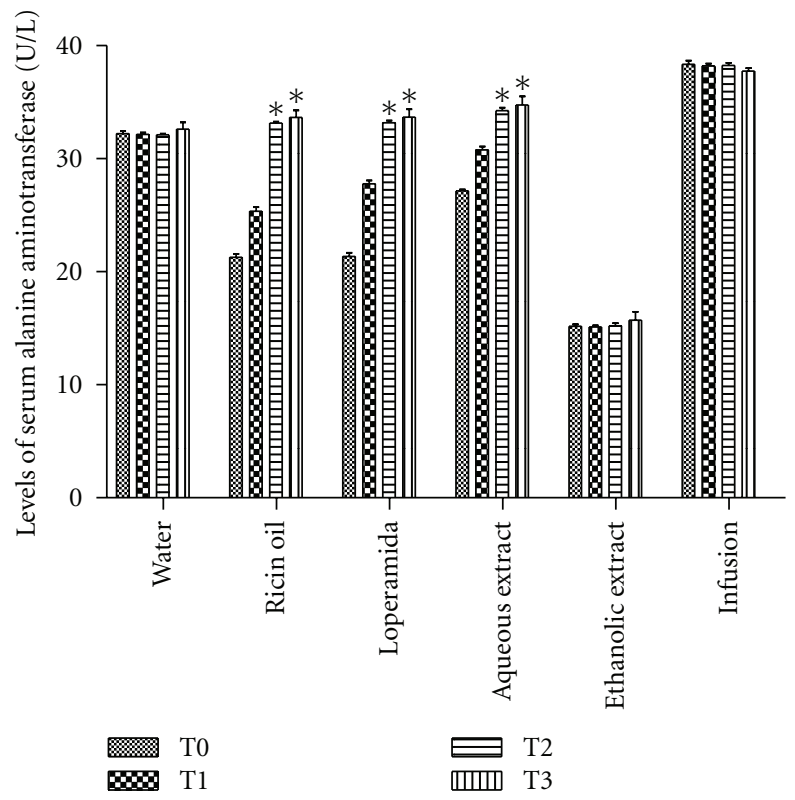

FIGURE 7: Evaluation of hepatotoxicity in rats after oral administration of infusion, ethanolic and aqueous extract of E. dysenterica utilizing as parameters alterations in the levels of serum (U/L) alanine aminotransferase (ALT) enzyme. Values of mean and standard deviation of the effect exerted by the samples at different periods: no treatments (T0), after one dose (T1), seven-day dose (T2) and 14-day dose (T3). Negative control group received $1 \mathrm{~mL}$ of water and ricin oil $\left(10 \mathrm{mg} \cdot \mathrm{kg}^{-1}\right)$. Positive control group received loperamide $\left(2 \mathrm{mg} \cdot \mathrm{kg}^{-1}\right)$ and experimental groups received infusion and aqueous extract at the dose of $800 \mathrm{mg} \cdot \mathrm{kg}^{-1}$ and ethanolic extract at the dose of $400 \mathrm{mg} \cdot \mathrm{kg}^{-1}$. Vertical bars represent standard error. Asterisks indicate statistical difference from control $(P \leq .05)$ by Dunnett test. 


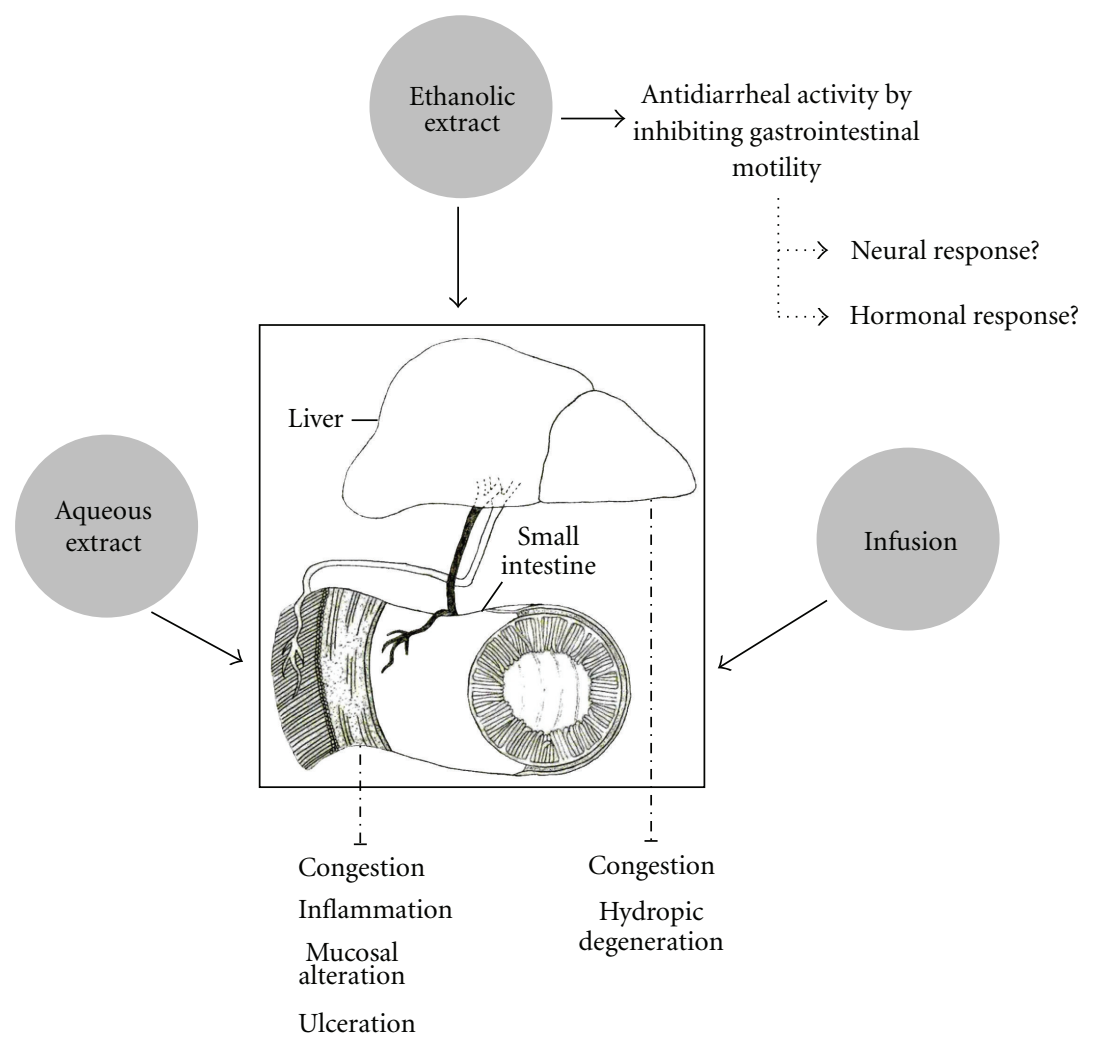

FIGURE 8: Hypothetical diagram summarizing the effect of ethanolic and aqueous extracts and infusion of E. dysenterica on the small intestine and liver.

[28]. Abascal and Yarnell in [29] comment on the tendency to use plants for treatment of irritable bowel syndrome (IBS). These authors describe the use of bayberry (Morella cerifera) root bark, meadowsweet (Filipendula ulmaria) leaf and cranesbill (Geranium maculatum) root; these plants all produce tannins that bind the fluid in the colon, inhibiting the protective excretory function of diarrhea. Antidiarrheal activity is also described in the model of secretory diarrhea induced by cholera toxin, after ingestion of a mixture of SP303 with procyanidin oligomers derived from the latex of Croton lechleri, known as Sangre de Drago [30].

The antidiarrheal activity of these compounds has been attributed to antimotility and antisecretory effects, and/or antimicrobial action [31]. In general, the presence of secretory diarrhea results in various electrolyte disorders. In the present study it was observed that the ethanolic extract of E. dysenterica causes electrolytic disturbance (affecting levels of chloride, magnesium, and phosphorus). Ion concentration analyses suggest that the antidiarrheal action was not caused by an antisecretory mechanism in the diarrhea model induced by ricin oil. It is known that ricin oil causes secretory diarrhea, since ricinoleic acid induces diarrhea through a hypersecretory response [32, 33]. Ricin oil reduces $\mathrm{Na}^{+}$and $\mathrm{K}^{+}$absorption and decreases $\mathrm{Na}^{+}$and $\mathrm{K}^{+}$ ATPase activity. These actions, related to ricin oil's diarrheal effects, are involved in adenylate cyclase activation, secretion of mucosal cAMP, induction of platelet activating factor, increase in prostaglandin biosynthesis, and modification of nitric oxide concentration [12-34]. Although these numerous mechanisms have been proposed, the overall consensus is that ricin oil-induced diarrhea stimulates prostaglandin formation; consequently, a drug that decreases the synthesis of prostaglandins can be used in the treatment of diarrhea [14-34].

Kaysar and collaborators [35] reported that the lowest serum phosphate concentration is associated with laxative abuse, which occasions severe diarrhea, resembling that induced by ricin oil. This showed that after one dose, these samples probably have no antidiarrheal activity. Moreover, phosphorus concentration being affected by different factors, such as vitamin D overdoses and laxative abuse or enemas (which are also absorbed when given rectally), occasions severe diarrhea [36]. In general, when serum phosphorus levels decrease, reduction in urinary phosphorus excretion and stimulation of the 1-alpha hydroxylase enzyme in the kidneys were observed [37]. However, in the presence of hyperphosphatemia, as observed after prolonged use of all samples, including loperamide (positive control), a rapid increase in urinary excretion of phosphorus occurs, mediated by the serum phosphorus level, parathyroid hormone (PTH), and fibroblast growth factor 23 (FGF23) [38]. This probably explains why loperamide has no direct effect on absorption, since its action mechanism is related to interaction with the $\mu$-opioid receptor, prolonging intestinal transit [39]. 
Abnormalities of chloride, phosphorus, and magnesium homeostasis are common and can be associated with dehydration caused by diarrhea [40]. Other authors report that hypomagnesemia involves intestinal loss, malabsorption, and abnormality of vitamin D and parathyroid hormone metabolism [41, 42].

Several antidiarrheal present toxicity effects, and for this reason, we make available the action of the ethanolic and aqueous extracts and infusion of $E$. dysenterica on the small intestine and liver, which is explained in Figure 8. The histopathological analysis of the small intestine showed serious injury to intestinal villi in animals treated with the infusion, ricin oil, and loperamide. The lesions in the epithelium observed in animals treated with these samples, including hemorrhage (data not shown), are indicative of several possible mucosal injuries. Some authors suggest that intestinal hemorrhage is associated with a significant localized inflammatory response in the mucosa and serosa, which they attributed to intestinal endotoxemia $[43,44]$. The persistent evidence of inflammation in the colonic mucosa of animals treated with infusion, ricin oil, and loperamide showed that excessive diarrhea observed could be caused by changes in epithelial permeability. These modifications could be related to modifications in tight junction function, which are often observed in ulcerative colitis [45].

Histopathological analysis of the liver in animals treated with loperamide, ethanolic and aqueous extracts showed hydropic degeneration, sinusoidal congestion in the capillaries and portal/periportal oedema formation. Similar results were described by Vasconcellos and collaborators [46], using leaves of Capraria biflora. It should be noted that the liver is a major site for the biotransformation, accumulation and excretion of exogenous chemicals, and that damage in the nearby tissue indicates possible toxicity [47].

The most common cause of abnormal liver enzymes in the sick patient is secondary liver changes that arise from a primary nonhepatic disease [48]. It is important to understand the reactive nature of the liver in response to other disease conditions. In such reactive situations, the hepatopathy is not the primary hepatic disease [49], and as we have seen in this study, there is a positive correlation between liver damage and changes in serum ALT.

An interesting observation, which also causes concern, is that although the infusion showed no biological activity in the tests used here, it did, however, present severe damage to the intestinal tissue and liver. These findings are worrying, since the Cerrado population frequently uses tea made from E. dysenterica leaves in the treatment of diarrhea.

In summary, data reported here demonstrated antidiarrheal activity by inhibiting gastrointestinal motility in animal models. Due to these findings, we believe that $E$. dysenterica could be a potential source of a new antidiarrheal drug, despite the above-mentioned toxicological effects. Results presented here confirm the popular use of E. dysenterica and its well known medical properties in folklore medicines. These data are extremely important, since the use of medicinal plants has recently increased in developed countries because of disillusionment with traditional medicine. In developing countries, the strategy of alternative medicine continues to be used due to low costs and abundant availability. The contribution of traditional knowledge can improve the development of more effective drugs with minimal or no side effects, also helping to conserve biological resources.

\section{Abbreviations}

5-HT4: 5-hydroxytryptamine (serotonin) receptor 4

ALT: Alanine aminotransferase

DMF: Dimethylformamide

FGF23: Fibroblast growth factor 23

HPLC: High-performance liquid chromatography

IBS: Irrifigure bowel syndrome

$\mathrm{mEq} / \mathrm{L}$ : Milliequivalents per liter

PTH: Parathyroid hormone

T0: $\quad$ No administration

T1: One dose administration

T2: $\quad$ Consecutive dose for seven days

T3: $\quad$ Repeated dose for fourteen days

TFA: Trifluoroacetic acid

WHO: World Health Organization.

\section{Acknowledgments}

The authors would like to thank the Brazilian Government, EMBRAPA, UCB, UFJF, CAPES, and CNPq.

\section{References}

[1] C. J. L. Murray and A. D. Lopez, "Mortality by cause for eight regions of the world: global burden of disease study," The Lancet, vol. 349, no. 9061, pp. 1269-1276, 1997.

[2] J. C. Teixeira and L. Heller, "Impact of water supply, domiciliary water reservoirs and sewage on faeco-orally transmitted parasitic diseases in children residing in poor areas in Juiz de Fora, Brazil," Epidemiology and Infection, vol. 134, no. 4, pp. 694-698, 2006.

[3] M. Field, "Intestinal ion transport and the pathophysiology of diarrhea," Journal of Clinical Investigation, vol. 111, no. 7, pp. 931-943, 2003.

[4] J. K. Anastasi, N. C. Dawes, and Y. M. Li, "Diarrhea and human immunodeficiency virus: a possible synergy for clinical practice," Journal of Alternative and Complementary Medicine, vol. 3, no. 2, pp. 163-168, 1997.

[5] V. Theodorou, M. Chovet, H. Eutamene et al., "Antidiarrhoeal properties of a novel sigma ligand (JO 2871) on toxigenic diarrhoea in mice: mechanisms of action," Gut, vol. 51, no. 4 , pp. 522-528, 2002.

[6] S. Brijesh, P. G. Daswani, P. Tetali, S. R. Rojatkar, N. H. Antia, and T. J. Birdi, "Studies on Pongamia pinnata (L.) Pierre leaves: understanding the mechanism(s) of action in infectious diarrhea," Journal of Zhejiang University Science B, vol. 7, no. 8, pp. 665-674, 2006.

[7] J. Jacobs, B. L. Guthrie, G. A. Montes et al., "Homeopathic combination remedy in the treatment of acute childhood diarrhea in Honduras," Journal of Alternative and Complementary Medicine, vol. 12, no. 8, pp. 723-732, 2006.

[8] A. Z. Kapikian, Y. Hoshino, R. M. Chanock, and I. PerezSchael, "Jennerian and modified Jennerian approach to 
vaccination against rotavirus diarrhea using a quadrivalent rhesus rotavirus (RRV) and human-RRV reassortant vaccine," Archives of Virology, Supplement, vol. 12, pp. 163-175, 1996.

[9] F. H. F. Galvão, D. L. Waitzberg, and E. Lima-Golçaves, "Permeabilidade intestinal de intestino delgado de ratos, preservados hipotermicamente com as soluções da Universidade de Winsconsin e Euro-collins," Acta Cirúrgica Brasileira, vol. 8, pp. 54-58, 1993.

[10] I.-M. Chang, "Initiative for developing evidence-based standardization of traditional Chinese medical therapy in the Western pacific region of the World Health Organization," Evidence-Based Complementary and Alternative Medicine, vol. 1, pp. 337-341, 2004.

[11] P. A. Nwafor, F. K. Okwuasaba, and L. G. Binda, "Antidiarrhoeal and antiulcerogenic effects of methanolic extract of Asparagus pubescens root in rats," Journal of Ethnopharmacology, vol. 72, no. 3, pp. 421-427, 2000.

[12] S. Afroz, M. Alamgir, M. T. H. Khan, S. Jabbar, N. Nahar, and M. S. K. Choudhuri, "Antidiarrhoeal activity of the ethanol extract of Paederia foetida Linn. (Rubiaceae)," Journal of Ethnopharmacology, vol. 105, no. 1-2, pp. 125-130, 2006.

[13] R. Rouf, S. J. Uddin, J. A. Shilpi, and M. Alamgir, "Assessment of antidiarrhoeal activity of the methanol extract of Xylocarpus granatum bark in mice model," Journal of Ethnopharmacology, vol. 109, no. 3, pp. 539-542, 2007.

[14] A. M. Mujumdar, A. V. Misar, and A. S. Upadhye, "Antidiarrhoeal activity of ethanol extract of the bark of Dalbergia lanceolaria," Journal of Ethnopharmacology, vol. 102, no. 2, pp. 213-216, 2005.

[15] C. G. Garcia, A. S. Polo, and N. Y. Murakami Iha, "Photoelectrochemical solar cell using extract of Eugenia jambolana Lam as a natural sensitizer," Anais da Academia Brasileira de Ciencias, vol. 75, no. 2, pp. 163-165, 2003.

[16] T. B. Lima, O. N. Silva, J. T. Oliveira et al., "Dentification of E. dysenterica laxative peptide: a novel strategy in the treatment of chronic constipation and irritable bowel syndrome," Peptides, vol. 31, no. 8, pp. 1426-1433, 2010.

[17] A. W. Ellery, "Guidelines for specification of animals and husbandry methods when reporting the results of animal experiments. Working committee for the biological characterization of laboratory animals/GV-solas," Laboratory Animals, vol. 19, no. 2, pp. 106-108, 1985.

[18] A. L. Abdullahi, M. O. Agho, S. Amos, K. S. Gamaniel, and C. Wambebe, "Antidiarrhoeal activity of the aqueous extract of Terminalia avicennoides roots," Phytotherapy Research, vol. 15, no. 5, pp. 431-434, 2001.

[19] L. Sagar, R. Sehgal, and S. Ojha, "Evaluation of antimotility effect of Lantana camara $\mathrm{L}$. var. acuelata constituents on neostigmine induced gastrointestinal transit in mice," BMC Complementary and Alternative Medicine, vol. 5, article 18, 2005.

[20] H. O. C. Mbagwu and O. O. Adeyemi, "Anti-diarrhoeal activity of the aqueous extract of Mezoneuron benthamianum Baill (Caesalpiniaceae)," Journal of Ethnopharmacology, vol. 116, no. 1, pp. 16-20, 2008.

[21] J. A. Ojewole, E. O. Awe, and W. D. Chiwororo, "Antidiarrhoeal activity of Psidium guajava Linn. (Myrtaceae) leaf aqueous extract in rodents," Journal of Smooth Muscle Research, vol. 44, no. 6, pp. 195-207, 2008.

[22] A. M. Mujumdar, A. V. Misar, and A. S. Upadhye, "Antidiarrhoeal activity of ethanol extract of the bark of Dalbergia lanceolaria," Journal of Ethnopharmacology, vol. 102, no. 2, pp. 213-216, 2005.
[23] S. Afroz, M. Alamgir, M. T. H. Khan, S. Jabbar, N. Nahar, and M. S. K. Choudhuri, "Antidiarrhoeal activity of the ethanol extract of Paederia foetida Linn. (Rubiaceae)," Journal of Ethnopharmacology, vol. 105, no. 1-2, pp. 125-130, 2006.

[24] S. Hughes, N. B. Higgs, and L. A. Turnberg, "Antidiarrhoeal activity of loperamide: studies of its influence on ion transport across rabbit ileal mucosa in vitro," Gut, vol. 23, no. 11, pp. 974-979, 1982.

[25] M. A. Khan, N. A. Khan, I. A. Qasmi, G. Ahmad, and S. Zafar, "Protective effect of Arque-Ajeeb on acute experimental diarrhoea in rats," BMC Complementary and Alternative Medicine, vol. 4, article no. 8, 2004.

[26] S. O. Aniagu, L. G. Binda, F. C. Nwinyi et al., "Anti-diarrhoeal and ulcer-protective effects of the aqueous root extract of Guiera senegalensis in rodents," Journal of Ethnopharmacology, vol. 97, no. 3, pp. 549-554, 2005.

[27] A. J. Akindele and O. O. Adeyemi, "Evaluation of the antidiarrhoeal activity of Byrsocarpus coccineus," Journal of Ethnopharmacology, vol. 108, no. 1, pp. 20-25, 2006.

[28] H. R. Chitme, R. Chandra, and S. Kaushik, "Studies on antidiarrheal activity of Calotropis gigantea R. Br. in experimental animals," Journal of Pharmacy and Pharmaceutical Sciences, vol. 7, no. 1, pp. 70-75, 2004.

[29] K. Abascal and E. Yarnell, "Combining herbs in a formula for irritable bowel syndrome," Alternative and Complementary Therapies, vol. 11, no. 1, pp. 17-23, 2005.

[30] K. Jones, "Review of sangre de drago (Croton lechleri)a South American tree sap in the treatment of diarrhea, inflammation, insect bites, viral infections, and wounds: traditional uses to clinical research," Journal of Alternative and Complementary Medicine, vol. 9, no. 6, pp. 877-896, 2003.

[31] M. J. G. Farthing, "Novel targets for the pharmacotherapy of diarrhoea: a view for the millennium," Journal of Gastroenterology and Hepatology, vol. 15, supplement, pp. G38-G45, 2000.

[32] H. V. Ammon, P. J. Thomas, and S. F. Phillips, "Effects of oleic and ricinoleic acids on net jejunal water and electrolyte movement. Perfusion studies in man," Journal of Clinical Investigation, vol. 53, no. 2, pp. 374-379, 1974.

[33] J. J. Stewart, T. S. Gaginella, and P. Bass, "Actions of ricinoleic acid and structurally related fatty acids on the gastrointestinal tract. I. Effects of smooth muscle contractility in vitro," Journal of Pharmacology and Experimental Therapeutics, vol. 195, no. 2, pp. 347-354, 1975.

[34] F. G. Shoba and M. Thomas, "Study of antidiarrhoeal activity of four medicinal plants in castor-oil induced diarrhoea," Journal of Ethnopharmacology, vol. 76, no. 1, pp. 73-76, 2001.

[35] I. I. Mahmoud, M. S. A. Marzouk, F. A. Moharram, M. R. ElGindi, and A. M. K. Hassan, "Acylated flavonol glycosides from Eugenia jambolana leaves," Phytochemistry, vol. 58, no. 8, pp. 1239-1244, 2001.

[36] N. Kaysar, J. Kronenberg, M. Polliack, and B. Gaoni, "Severe hypophosphataemia during binge eating in anorexia nervosa," Archives of Disease in Childhood, vol. 66, no. 1, pp. 138-139, 1991.

[37] Y. Beloosesky, J. Grinblat, A. Weiss, B. Grosman, U. Gafter, and A. Chagnac, "Electrolyte disorders following oral sodium phosphate administration for bowel cleansing in elderly patients," Archives of Internal Medicine, vol. 163, no. 7, pp. 803-808, 2003.

[38] S. M. Moe, "Disorders involving calcium, phosphorus, and magnesium," Primary Care, vol. 35, no. 2, pp. 215-237, 2008. 
[39] A. De Luca and I. M. Coupar, "Insights into opioid action in the intestinal tract," Pharmacology and Therapeutics, vol. 69, no. 2, pp. 103-115, 1996.

[40] S. A. Kiss, T. Forster, and Á. Dongó, "Absorption and effect of the magnesium content of a mineral water in the human body," Journal of the American College of Nutrition, vol. 23, no. 6, pp. 758S-762S, 2004.

[41] W. M. T. Groenestege, S. Thébault, J. van der Wijst et al., "Impaired basolateral sorting of pro-EGF causes isolated recessive renal hypomagnesemia," Journal of Clinical Investigation, vol. 117, no. 8, pp. 2260-2267, 2007.

[42] S. E. Kimmel, L. S. Waddell, and K. E. Michel, "Hypomagnesemia and hypocalcemia associated with protein-losing enteropathy in Yorkshire Terriers: five cases (1992-1998)," Journal of the American Veterinary Medical Association, vol. 217, no. 5, pp. 703-706, 2000.

[43] D.-W. Han, "Intestinal endotoxemia as a pathogenetic mechanism in liver failure," World Journal of Gastroenterology, vol. 8, no. 6, pp. 961-965, 2002.

[44] Y. Shibayama, "An experimental study on the pathogenesis of acute haemorrhagic enteropathy-significance of congestion and endotoxaemia," Journal of Pathology, vol. 148, no. 2, pp. 169-174, 1986.

[45] V. Binder, L. Elsborg, and J. Greibe, "Disodium cromoglycate in the treatment of ulcerative colitis and Crohn's disease," Gut, vol. 22, no. 1, pp. 55-60, 1981.

[46] M. C. Vasconcellos, D. P. Bezerra, A. M. Fonseca et al., "Antitumor activity of biflorin, an o-naphthoquinone isolated from Capraria biflora," Biological and Pharmaceutical Bulletin, vol. 30, no. 8, pp. 1416-1421, 2007.

[47] M. Sharma, D. E. Shubert, M. Sharma et al., "Biotransformation of tamoxifen in a human endometrial explant culture model," Chemico-Biological Interactions, vol. 146, no. 3, pp. 237-249, 2003.

[48] S. Venkateswaran, L. Pari, P. Viswanathan, and V. P. Menon, "Protective effect of Livex, a herbal formulation against erythromycin estolate induced hepatotoxicity in rats," Journal of Ethnopharmacology, vol. 57, no. 3, pp. 161-167, 1997.

[49] V. Kumar, A. K. Abbas, J. Aster, and N. Fausto, Robbins and Cotran Pathologic Basis of Disease, Elsevier, Berlin, Germany, 2005. 


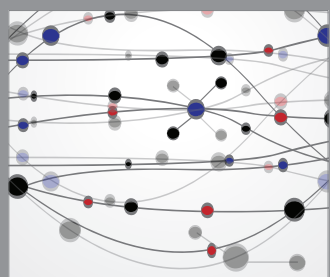

The Scientific World Journal
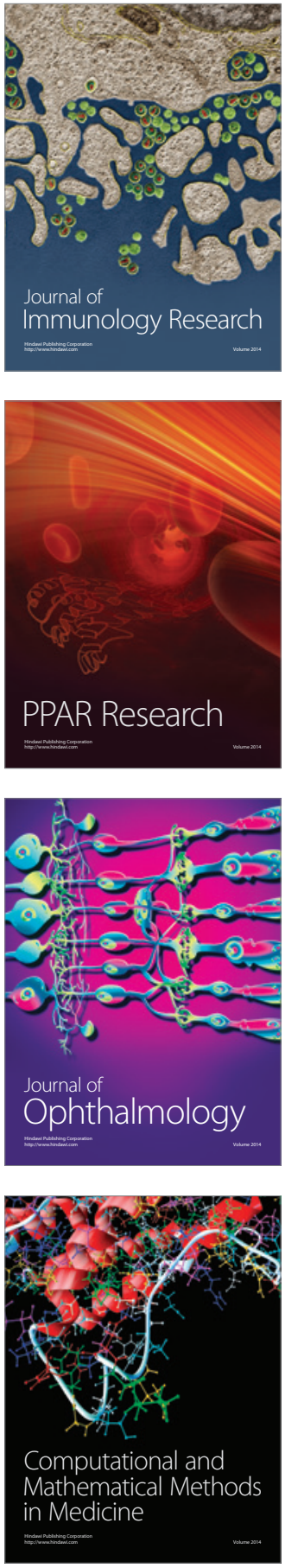

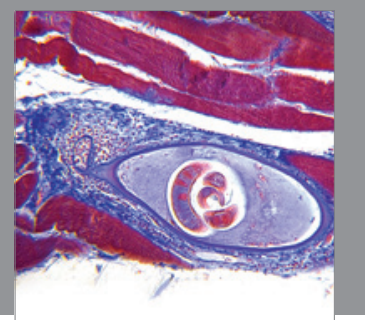

Gastroenterology

Research and Practice
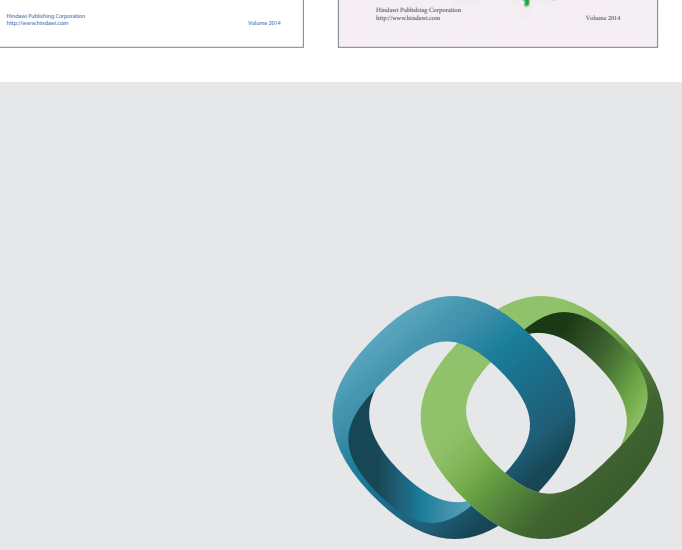

\section{Hindawi}

Submit your manuscripts at

http://www.hindawi.com
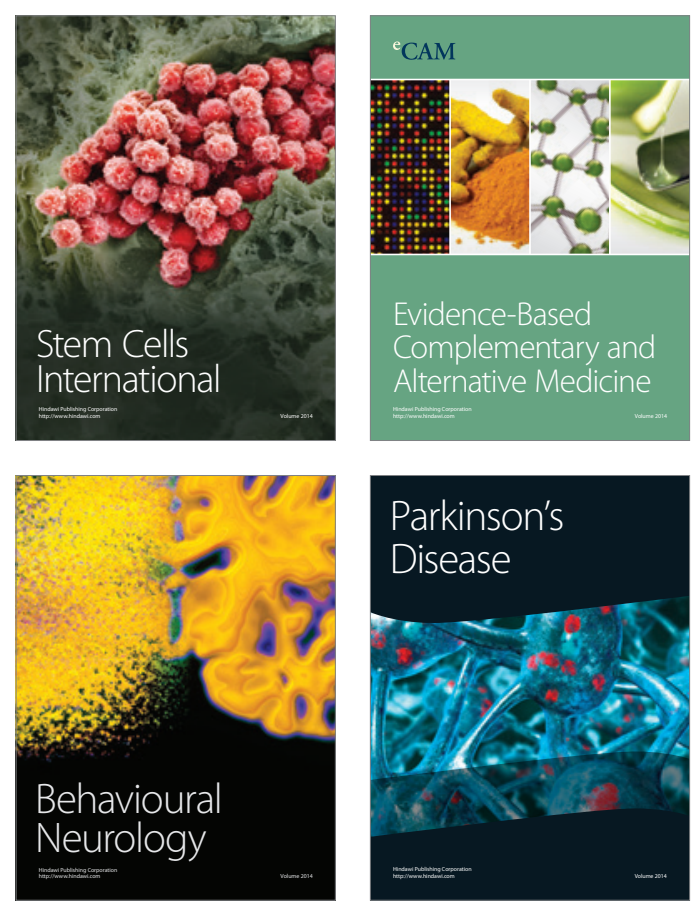

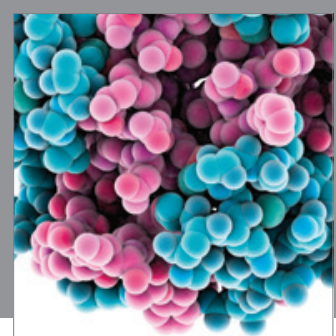

Journal of
Diabetes Research

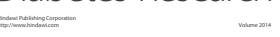

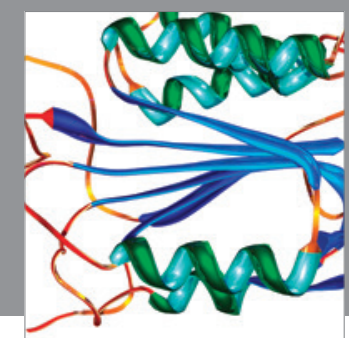

Disease Markers
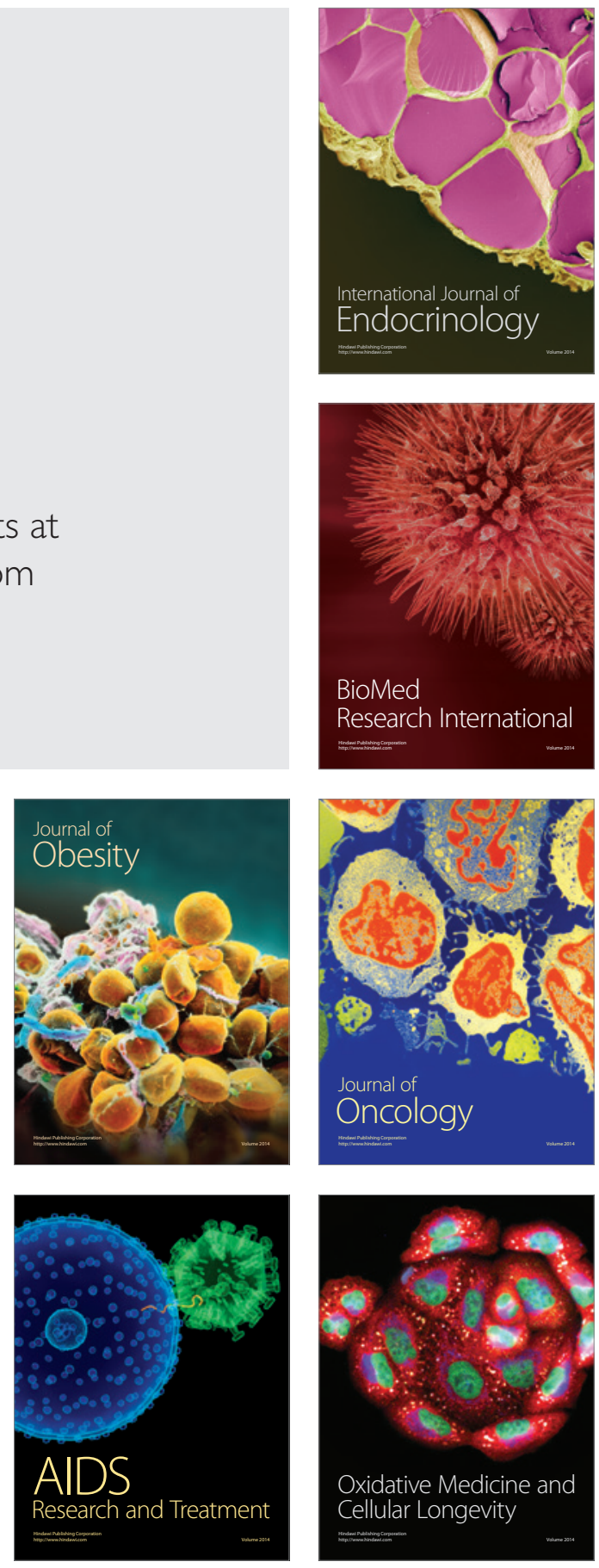